\title{
Real-time observations of tooth demineralization in 3 dimensions using $\mathrm{X}$-ray microtomography
}

\section{Short title: Real-time 3D tooth demineralization}

Graham Roy Davis, Professor of 3D X-ray Imaging

David Mills, X-ray microtomography facility manager

Paul Anderson, Professor of Oral Biology

Institute of Dentistry

Barts and the London School of Medicine and Dentistry

Queen Mary University of London

London E1 4NS

United Kingdom

Corresponding Author: Prof. Graham R Davis

Tel: +44 (0)20 78825967

Fax: +44 (0)2078827089

Email: G.R.Davis@qmul.ac.uk

Keywords: Microtomography; micro-CT; dental enamel; caries; demineralization 


\title{
Real-time Observation of tooth demineralization in 3 dimensions using $\mathrm{X}$-ray microtomography
}

\begin{abstract}
Objectives

The immediate aim of this study was to develop and test a method to record, visualize and quantify real-time demineralization (or remineralization) of teeth in vitro using $X$ ray microtomography (XMT or micro-CT). The longer term objective is to improve understanding of demineralization processes, allowing the creation of better artificial models of dental caries and better quantification of the efficacy of caries treatment and prevention regimes.
\end{abstract}

\section{Methods}

For demineralization studies, the tooth is mounted in a plastic container through which the demineralizing solution is circulated during simultaneous repeated scanning. Key features of the method are safe circulation of the demineralizing solution, periodic interruption to adjust X-ray filament current and re-focus, accurate beam-hardening correction and calibration, alignment of reconstructed scans, and normalization of greylevels to compensate for changes in the X-ray spectrum. The method was tested by scanning an extracted third molar during 8 days of demineralization.

\section{Results}

From the reconstructed 3D images, the progression of an artificial carious lesion could be visualized and quantified. The lesion progressed at approximately $0.1 \mathrm{~mm}$ per day and appeared to be more erosive in nature.

\section{Conclusions}

A successful method has been developed to monitor real-time demineralization in 3 dimensions. Further work is now needed to create better models of true carious lesions.

\section{Clinical Significance}

Observation of the dynamics of demineralization and remineralization will aid in the development of therapies to treat and prevent dental caries.

Keywords: Microtomography; micro-CT; dental enamel; caries; demineralization 


\section{Introduction}

The use of X-ray microtomography (XMT or micro-CT) is well established in dental research[1]. XMT has been shown to be an excellent tool to evaluate morphological features, such as changes in shape $[2,3]$ and the presence of voids $[4,5]$ in ex vivo studies of root canal instrumentation and obturation. With appropriate beamhardening correction and calibration, the technique can also be used to study dental hard tissue loss following acidic challenge, by quantification of mineral concentration [68]. Repeated scanning allows changes to be observed over time. Previously, this has been associated with very rapid scanning, such as for observing a beating heart [9]; it may be referred to as dynamic or 4D micro-CT. However, for slower processes, e.g. in vitro tooth mineral loss or gain experiments during acid attack, the scan time can be longer, allowing good quality images to be obtained with no special reconstruction algorithm. In order to do this, it is necessary to immerse the tooth in a circulating acid or remineralization solution throughout the scanning period, which may be several days, or even weeks. In terms of hardware, the main consideration is ensuring that there is no risk of leakage within the scanner, especially since scans may be left unattended overnight. In addition to the usual tomographic reconstruction, processing involves aligning each reconstructed volume (to compensate for positional drift) and calibrating to allow for slight changes in the X-ray spectrum that may occur over time. Although synchrotron based micro-CT systems may be able to deliver higher quality images, their use for such slow dynamic processes is logistically impractical and thus only impact source based systems, with their inherent problems with beam-hardening and low flux, are suitable.

In the past, X-ray absorption studies to measure changes in tooth mineral have been carried out on 2D sections of enamel exposed to acid challenges[10]. This involves considerable destruction and therefore potential damage to the specimen, which could modify the progress of acid. Further, as the architecture of enamel is complex, reducing the progress to effectively two dimensions will not be a true reflection of the overall process.

The aim of this paper is to describe the hardware and software necessary for 3D realtime observation of tooth demineralization and to demonstrate this method with an 8day continuous scan of tooth mineral loss in an experimental acid challenge.

\section{Method}

\subsection{Scanner}

The scanner used was the MuCAT 2[11] system developed at Queen Mary University of London, employing time-delay integration (TDI) to avoid ring artefacts[12] and facilitate high signal-to-noise ratio imaging. In this case, the relatively short scan times meant that photon statistics were the primary limitation on image quality and thus a 
conventional X-ray imaging system could have been used, and indeed may have been more efficient. The TDI system does, however, have the advantage that it does not suffer from burn-in artefacts caused by regional variations in scintillator light output that change over time according to exposure.

The X-ray generator and enclosure was an HMX 225 system (Nikon Metrology, UK) which, for the experiment described, was operated at $90 \mathrm{kV}$ and $180 \mu \mathrm{A}$, with an internal air temperature stabilization system set to $22^{\circ} \mathrm{C}$. The X-ray enclosure was of sufficient size to allow the circulation system to be housed internally, thus maintaining the temperature of the circulating solution. Note, an alternative would be to run tubing though the cable labyrinth to allow the pump to be mounted outside the X-ray enclosure.

An 800 S series camera (Spectral Instruments, Tucson, Arizona) with a 16 megapixel Fairchild CCD485 detector was used, coupled to a Csl scintillator. For TDI readout, this is mounted on a horizontal linear stage that moves the camera through the X-ray shadow at a velocity synchronized to the CCD readout.

\subsection{Circulation}

One litre of standard acetic acid demineralizing solution was prepared at $\mathrm{pH}=4.0$ using analytical grade reagents[13]. A large volume was used to prevent the accumulation of dissolution products reducing the reaction kinetics. To minimize the risk of spills within the X-ray enclosure, a "double-safe" pumping method was used (Figure 1). The first principle of this is that the circulating solution is drawn through the specimen container by the pump from the reservoir (consisting of a 1 litre bottle with two holes drilled in the cap for the tubes and a further hole to prevent changes in pressure). This creates a negative relative pressure within the circulation tubes and specimen container, whereby a small leak would result in air being drawn into the system and a major leak would simply stop the circulation altogether. The second principle in the double-safe methodology is that the solution is drawn up through a tube that protrudes just below the surface in the reservoir. If for any unforeseen reason, the solution should leak into the cabinet, the reservoir level would drop and circulation would cease. The pump and reservoir were themselves placed in a large plastic tray, providing a further level of protection. PVC tubing of $2.79 \mathrm{~mm}$ inner diameter [Altek, UK] was used, except through the peristaltic pump, which used silicone tubing [Altek, UK] with an inner diameter of $4 \mathrm{~mm}$. The tubes to and from the specimen container were passed through an overhead guide, with sufficient slack to allow them to twist through $360^{\circ}$ throughout the course of a single scan. The scanner software was configured to "rewind" the specimen rotation stage after each scan. 


\subsection{Specimen preparation}

A caries free third molar was selected from a human tissue bank; ethical approval was obtained from the Queen Mary Research Ethics Committee (QMREC2008/57). The tooth was then coated with acid resistant nail varnish, except for a $5 \mathrm{~mm}$ window on the buccal surface, as is done in conventional tooth mineral loss microradiographic studies[14]. The tooth was then secured in the XMT circulation cell using soft wax (6969 from Poth Hille \& Co Ltd, Rainham, Essex, UK) around the roots.

\subsection{Procedure}

The X-ray source was of the demountable type, with a filament lifetime of around two weeks of continuous use. However, throughout its lifetime, the filament becomes thinner and requires less current to maintain its temperature. The lifetime is considerably reduced if the current is not reduced throughout its lifetime. There is also a need to change the reservoir solution periodically to remove reaction products. For these reasons, scanning was briefly interrupted approximately every 24 hours to reduce the filament current to the minimum level that provided an acceptable focus and to refresh the reservoir; this took approximately 10 minutes. A calibration carousel was also scanned at the end of each 24-hour period[15]. The voxel size was set to $30 \mu \mathrm{m}$, with 927 projections around $360^{\circ}$, taking 2.6 hours, allowing 9 scans per day. The first scan began as soon as the circulating solution had filled the specimen container. After four 24 hour periods, the scans were set to run continuously for four days to cover an un-attended period (there was less concern over filament failure at the end of the experiment when sufficient data would have been collected). In total, 73 scans were completed.

\subsection{Processing}

The calibration carousel scans ( 9 projections) were used to optimize a model of the Xray spectrum which was then used to create a $2 \mathrm{D}$ correction transform for beamhardening correction[16]. This is a sophisticated correction method that considers both the amount of mineral in the beam and "organic" material (including biological tissue, circulating solution and plastic specimen container). The $90 \mathrm{kV}$ polychromatic projections were transformed to $40 \mathrm{keV}$ equivalent monochromatic projections. Tomographic reconstruction was performed using Feldkamp back-projection[17] to create the 73 volume images. The first image in the sequence was used as a reference and the remaining ones were aligned to this using in-house alignment software with 7 degrees of freedom ( $x-, y$ - and $z$-shift, $x_{-}, y_{-}$, and z-rotation and isotropic scale). This was developed prior to, but is similar in principle to a published method[18], which could serve the same purpose (though with only 6 degrees of freedom). The method used here, however, based its alignment on edge enhanced images, which is less prone to being skewed by the moving lesion front). Because of the fixed position of the specimen, only very minor corrections were required, facilitating fully automatic 
alignment (where the initial misalignment is large, approximate manual alignment is first required).

Using in-house "Tomview" software, a set of tags (coordinate markers) were positioned within the final scan, identifying regions in enamel and dentine away from demineralized regions that could be used as references for mineral concentration. Using the defined tag coordinates, the linear attenuation coefficient (LAC) was measured within a 3-voxel radius of each tagged point in the first scan and the mean of all these values was used as a reference value. The same mean was measured for the remaining scans and the greyscale values (LAC) were multiplied by an appropriate factor to match the reference scan.

\subsection{Analysis}

In order to visualize and analyze the lesion formation, each corrected scan was subtracted from the baseline scan (at time zero). The resultant 3D images were then cropped (by the same amount) to the minimal cuboidal volume that enclosed the lesion. To convert to mineral concentration, the following formula was used:

Where:

$$
c=\frac{\mu-\mu_{0}}{\mu_{m}-\mu_{s}} \rho_{m}
$$

$\mu-\mu_{0}$ is the difference in the measured LAC

$\mu_{m}$ is the known LAC of $100 \%$ mineral

$\mu_{s}$ is the mean measured LAC of the solution (assumed to be approximately the same as the organic component of the tooth tissue.

$\rho_{m}$ is the known density of $100 \%$ mineral

The mineral was assumed to be calcium hydroxyapatite with a density of $3.16 \mathrm{gcm}^{-3}$ and an LAC of $3.12 \mathrm{~cm}^{-1}$ at $40 \mathrm{keV}$ from the XCOM database[19].

The lesion was defined by setting a threshold in the difference scans of $10 \%$ of the mean enamel LAC (representing a $10 \%$ drop in mineral concentration). The number of voxels above the threshold was used to calculate the volume. The lesion depth was taken as the largest sphere that could fit within the lesion. This was calculated as the maximum of the thickness transform of the defined lesion [20]. 


\section{$3 \quad$ Results}

Results here are presented as verification of the methodology; a full study of the demineralization of this and other samples (including other imaging modalities) will be the subject of a future publication. The experiment ran to completion with no detectable leakage of the circulating solution or unscheduled interruption of the X-ray beam.

\subsection{Alignment of reconstructed volumes.}

The alignment software recorded a shift of 2.0, 1.0 and -0.9 voxels in the $x, y$ and $z$ directions respectively between the first and last scan. These shifts represent drift distances of 60,30 and $-27 \mu \mathrm{m}$ respectively. There was no detectable angular shift in any direction, nor change in scale.

\subsection{Grey level correction}

Between calibrations, there was an apparent drop in the grey level (LAC) of up to $2 \%$ over the 24 -hour periods and $3 \%$ over the final 4 days.

\subsection{Preliminary Analysis}

An artificial lesion was created in the windowed region of the tooth, reaching a depth of approximately $0.9 \mathrm{~mm}$. It appeared to advance at an approximately constant rate throughout the experiment (Figure 2[, Supplementary Video 1, Video 2, Video 3; note Video 3 was rendered using Drishti from the Australian National University] ${ }^{1}$ ). A line profile through the lesion on the last scan shows a plateau shape with a "sharp" transition on the inner and outer edges (Figure 3). From this figure, it can be seen that the progression of the lesion front is decelerating (note that for clarity, this figure only displays data from 1 in 18 of the available scans). The LAC of the enamel away from the lesion is around $2.89 \mathrm{~cm}^{-1}$ at the outer surface, falling to $2.58 \mathrm{~cm}^{-1}$ near the enameldentine junction (EDJ). This corresponds to mineral concentrations of $2.90 \mathrm{~g} \mathrm{~cm}^{-3}$ and $2.56 \mathrm{~g} \mathrm{~cm}^{-3}$ respectively. Previous testing of the 2D beam-hardening correction technique[16] indicates that although dishing artefacts are greatly reduced, the absolute values may be slightly underestimated. Thus, these values are best interpreted in terms of their relative proportion of the mineral concentration in healthy enamel. At the outer edge of the lesion, there is no detectable difference in LAC between the former location of healthy enamel (prior to acid demineralization) and the circulating solution, indicating that all mineral has been lost. It is not possible, with this technique, to determine if any remaining organic structure exists. After 8 days, within the bulk of the lesion, the LAC had fallen to around $0.55 \mathrm{~cm}^{-1}$, corresponding to a mineral concentration of $0.30 \mathrm{~g} \mathrm{~cm}^{-3}$. This is around $10 \%$ of the concentration in sound enamel.

${ }^{1}$ Online version only 
The progression of the lesion, in terms of mass, volume and thickness are shown in Figure 4.

\section{Discussion}

Using the above method, the feasibility of monitoring real-time acid demineralization in 3D has been demonstrated. Although the method need not be duplicated exactly, the following are identified as key features:

1. Safe circulation of the demineralizing solution.

2. Continuous scanning with "rewind" of the rotation stage to prevent tube twisting.

3. Periodic interruption to adjust filament current and re-focus if using a demountable X-ray source.

4. Good beam-hardening correction (the method used here corrects for additional beam-hardening introduced by immersion).

5. Alignment of reconstructed 3D images to compensate for drift.

6. Normalization of reconstructed images to compensate for changes in the X-ray spectrum.

The pre-correction grey level drop of up to $2 \%$ in 24 hours is surprisingly high and exceeds that observed in previous experiments. A possible explanation is pitting in the X-ray target which caused the spectrum to change with even the slightest drift of the electron beam focus. The target system used allows the anode to be indexed (rotated on a screw thread), thus allowing a fresh area of the target to be exposed. In future, this indexing will be performed prior to the start of the experiment, although the repeated calibration and normalization process prevented this spectral drift from impacting the final results.

Alignment correction was necessary to precisely monitor the progression of the lesion front and to allow image subtraction in order to show the lost mineral. In an unaligned subtraction image (Figure 5), misalignment errors can be seen in the position of the EDJ and outer edge of the enamel (note, negative errors are not visible), demonstrating the need for alignment. The lack of changes in orientation or scale implies that a much simpler alignment algorithm could be used with only 3 degrees of freedom ( $x-, y-$ and $z-$ shift).

In this instance, the artificial lesion generated was more akin to acid erosion than caries formation, although examination of all the image data showed that some local partial surface layers exist during the development of the lesion. This made definition of the lesion a simple matter of thresholding and allowed a relatively simple measurement of depth using the thickness transform. All three measurements of lesion progression (Figure 4) follow a similar trajectory, except at the very early stage where the lesion is first forming. 
Future studies will include increasing the $\mathrm{pH}$ so that the lesion progression is slower, and cycling demineralization and remineralization to simulate the natural oral environment. Should this prove to be successful in creating more caries-like lesions with surface layers and more diffuse fronts, volume and depth will be more difficult to define.

Longer experiments are feasible, but of course demand continuous use of the scanner. Periods in excess of 2 weeks may need to be interrupted for the time taken to replace the filament. This is likely to be a minimum of 2 hours to allow pumping down and conditioning of the X-ray generator. Generators employing sealed sources would not be subject to this interruption.

\section{Conclusion}

The development of this methodology and the success of the feasibility study pave the way for a whole new area in research into the 3D dynamic processes involved in tooth demineralization and remineralization. In this preliminary analysis, only a fraction of the available data has been made use of. Further analysis will be performed, looking at more time points and studying both the advance and spread of the lesion. It is hoped that this method will increase our understanding of the formation of surface layers (a barrier to remineralization) and aid in the development of new remineralization paradigms. The methods described here can, of course, be used to study other dynamic processes that progress at similar rates.

\section{Acknowledgements:}

The authors would like to thank Huda Al-mandil for preparation of the sample. 


\section{Figures}

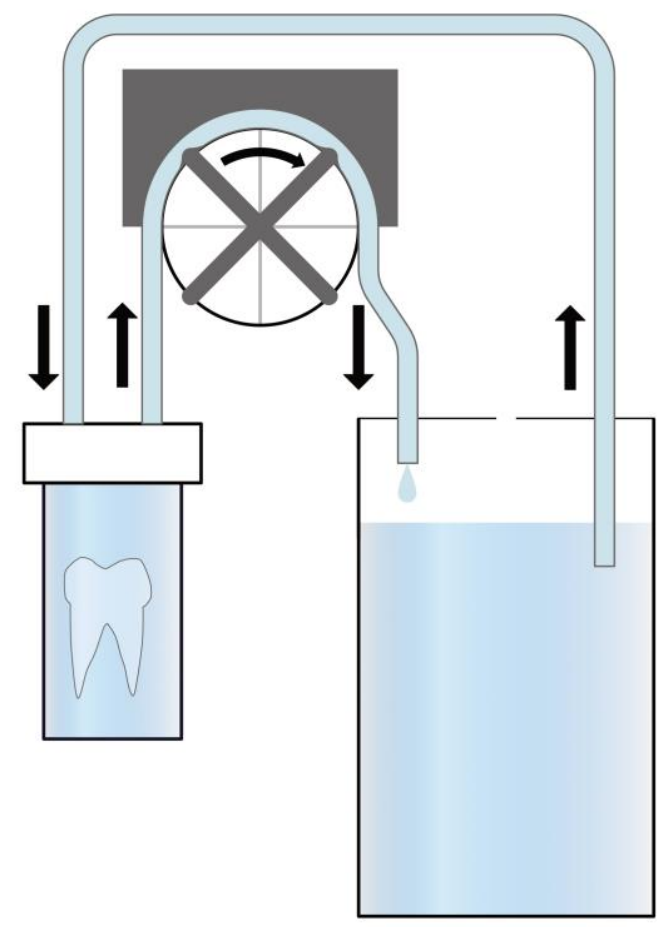

Figure 1: Double safe pumping principle. Demineralizing solution is drawn through the specimen from a tube descending just below the surface in the reservoir. 


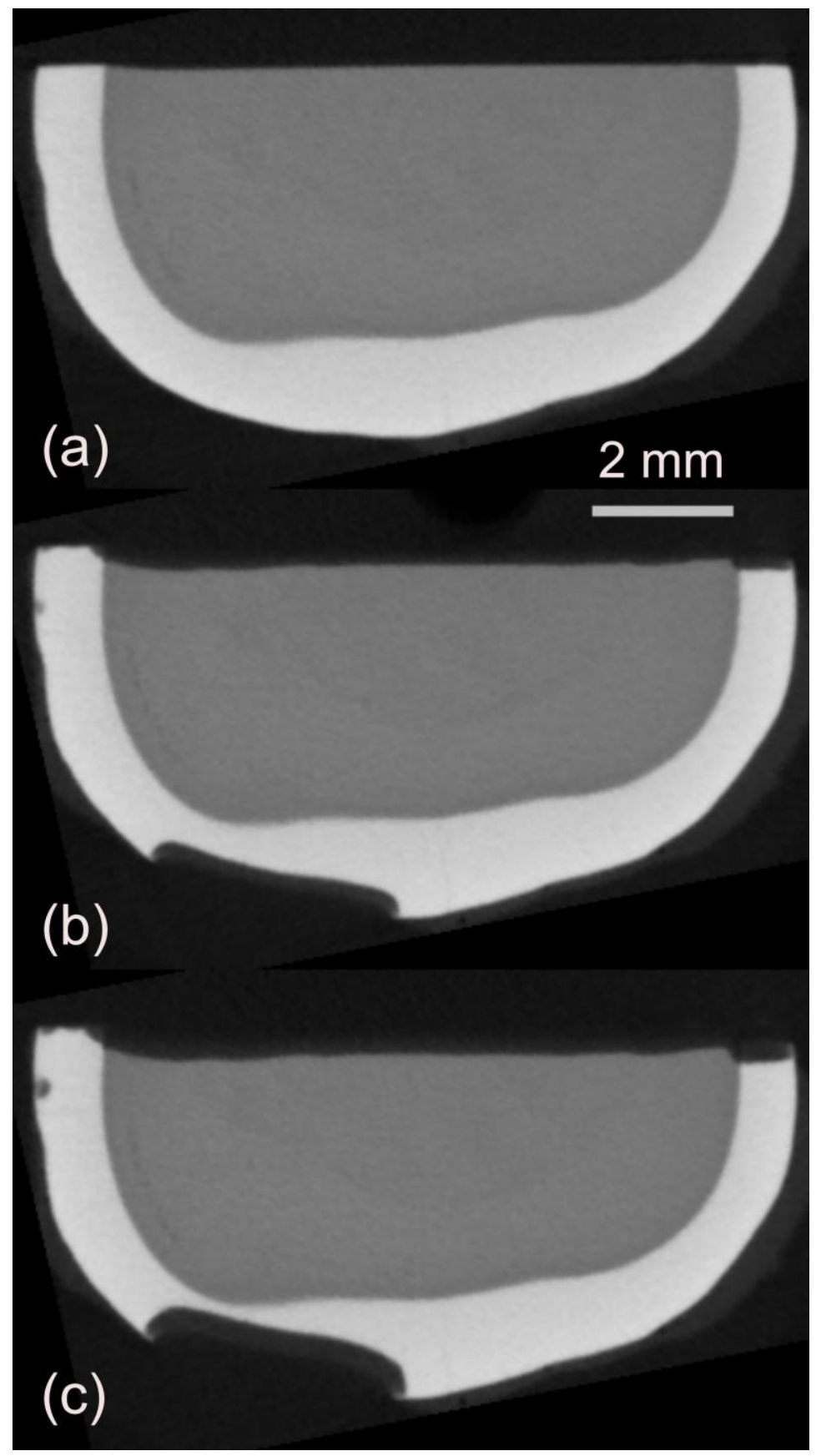

Figure 2: Double safe pumping principle. Demineralizing solution is drawn through the specimen from a tube descending just below the surface in the reservoir. 


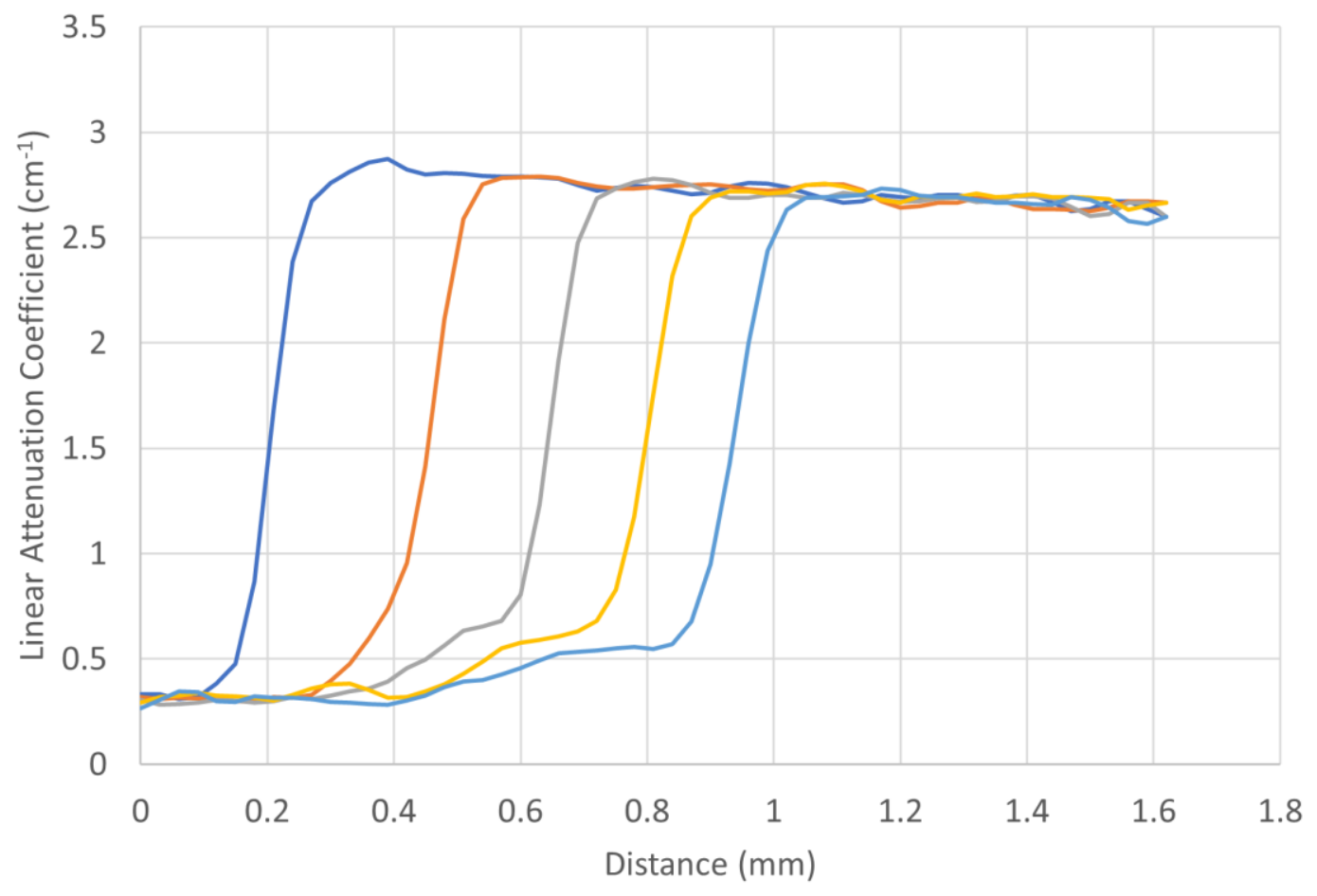

$-0 \mathrm{~d}-2 \mathrm{~d}-4 \mathrm{~d}-6 \mathrm{~d}-8 \mathrm{~d}$

Figure 3: Line profile through the lesion at 2-day intervals. 
Lesion Mass

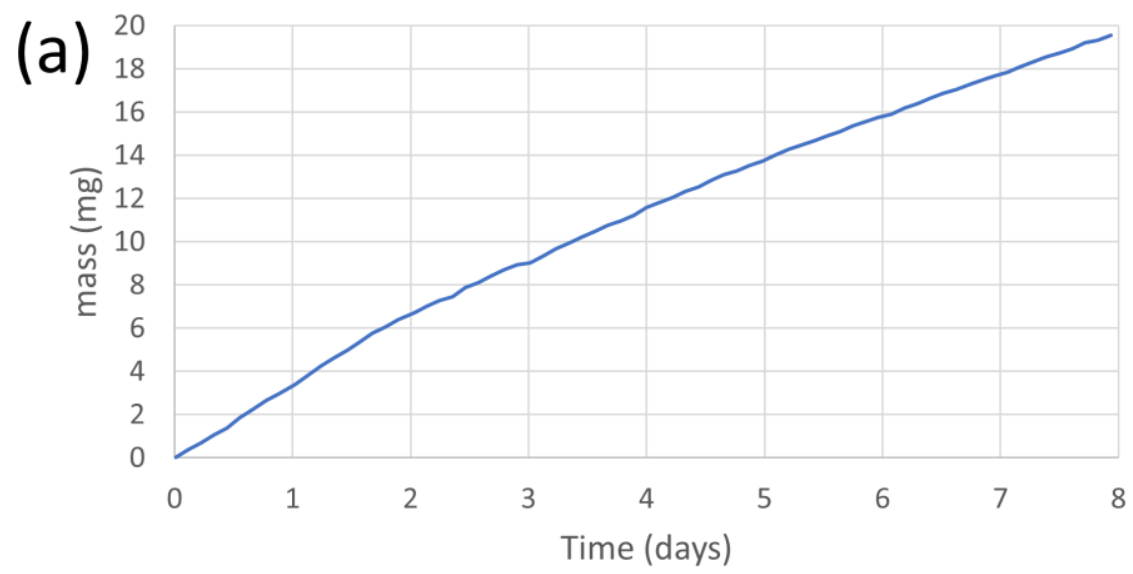

Lesion Volume

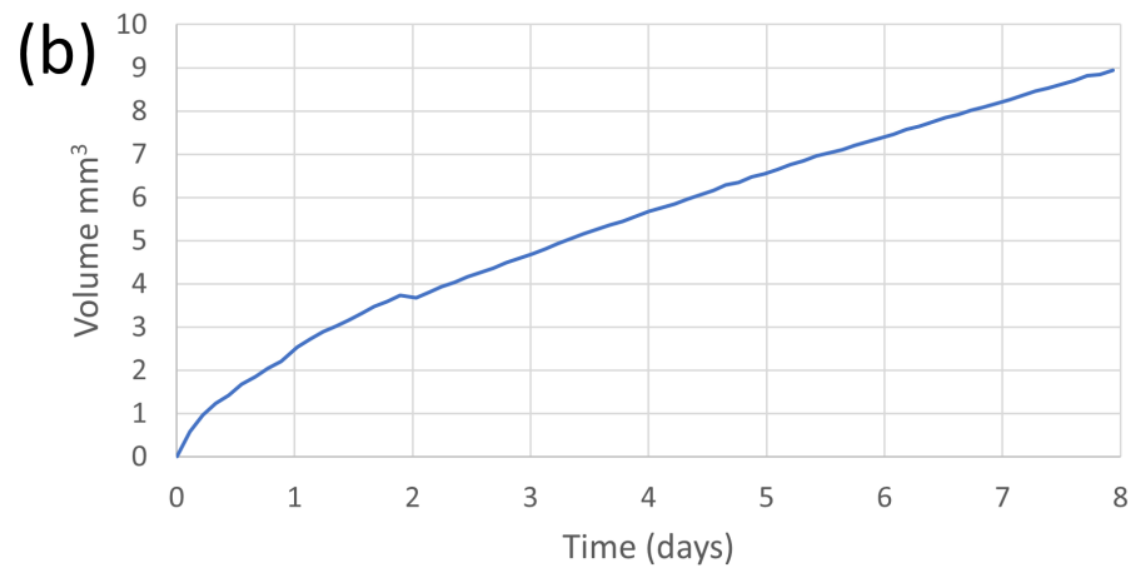

\section{Lesion Depth}

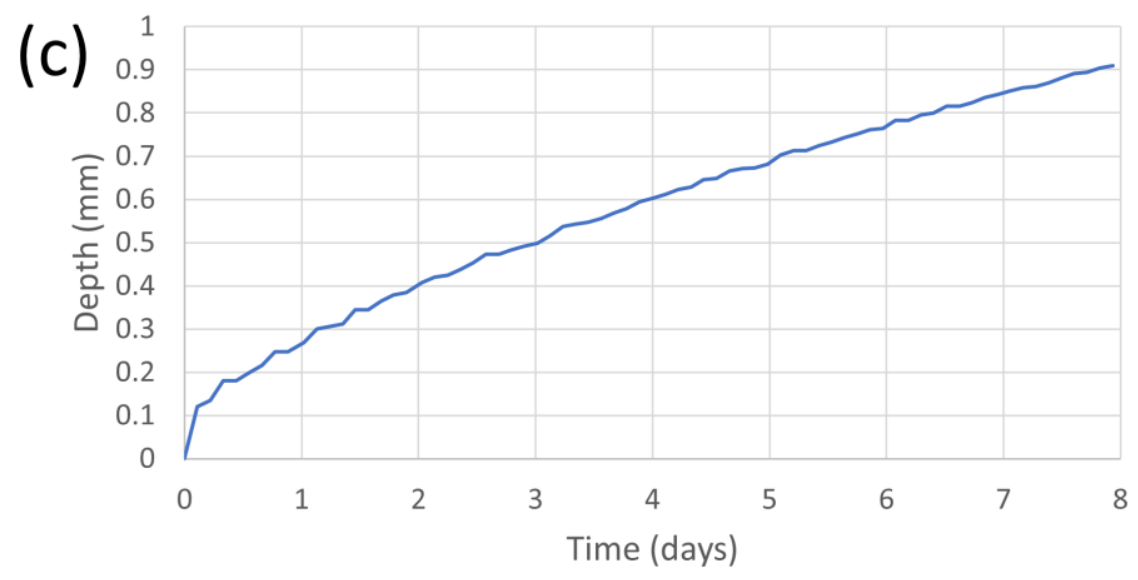

Figure 4: Plots of lesion growth vs time (2.6 hour intervals) in terms of (a) mass, (b) volume and (c) depth. 

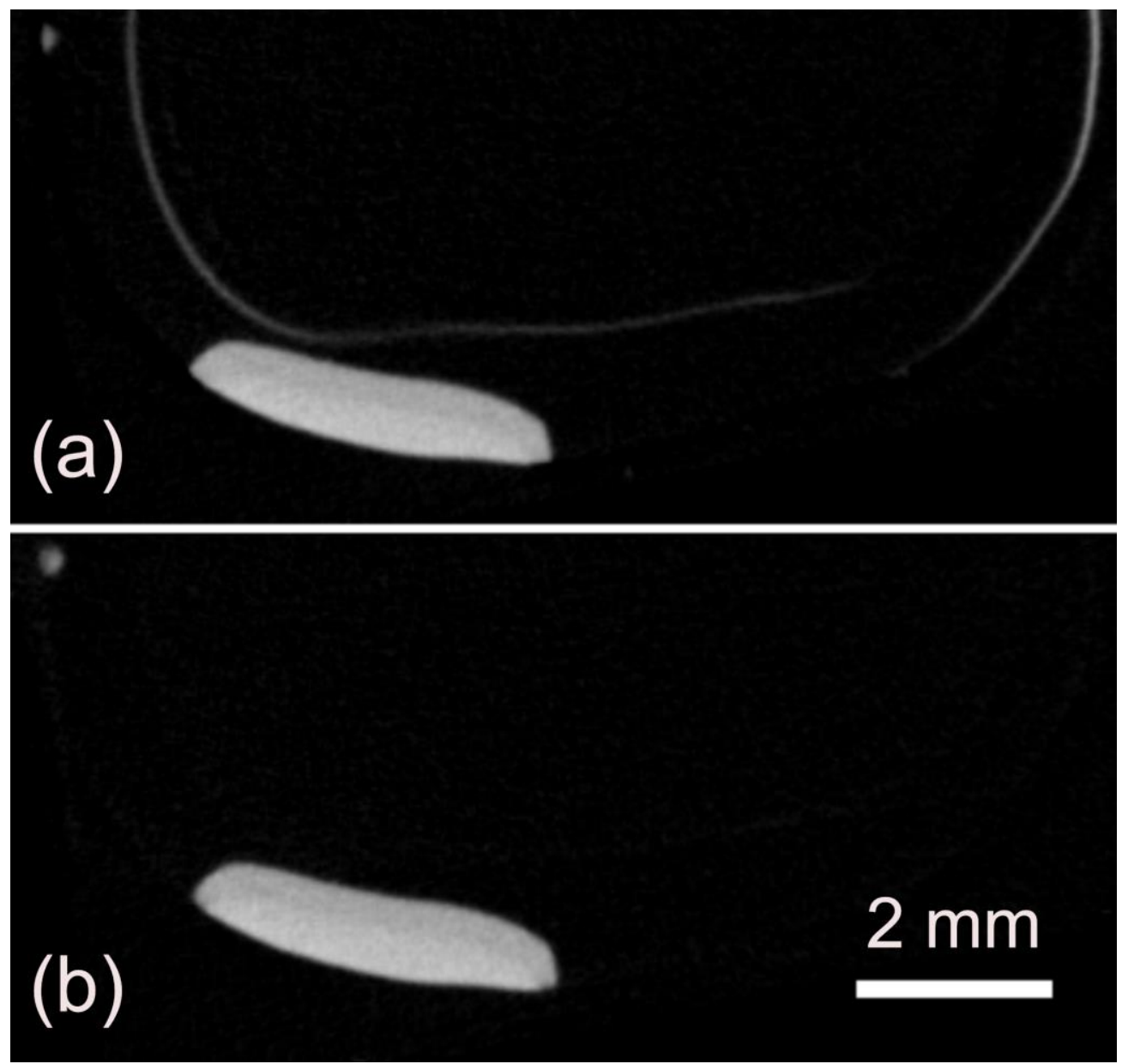

Figure 5: Slice through subtraction of the last from the first volume; (a) without alignment and (b) with alignment.

\section{References}

[1] M.V. Swain, J. Xue, State of the Art of Micro-CT Applications in Dental Research, Int J Oral Sci 1(4) (2009) 177-188.

[2] O.A. Peters, K. Schonenberger, A. Laib, Effects of four Ni-Ti preparation techniques on root canal geometry assessed by micro computed tomography, International endodontic journal 34(3) (2001) 221-30.

[3] O.A. Peters, C.I. Peters, K. Schonenberger, F. Barbakow, ProTaper rotary root canal preparation: effects of canal anatomy on final shape analysed by micro CT, International endodontic journal 36(2) (2003) 86-92.

[4] M. Jung, D. Lommel, J. Klimek, The imaging of root canal obturation using microCT, International endodontic journal 38(9) (2005) 617-626.

[5] M. Hammad, A. Qualtrough, N. Silikas, Evaluation of root canal obturation: a threedimensional in vitro study, J Endod 35(4) (2009) 541-4. 
[6] N.S. Willmott, F.S.L. Wong, G.R. Davis, An X-ray microtomography study on the mineral concentration of carious dentine removed during cavity preparation in deciduous molars, Caries Res 41(2) (2007) 129-134.

[7] M. Ahmed, G.R. Davis, F.S. Wong, X-Ray Microtomography Study to Validate the Efficacies of Caries Removal in Primary Molars by Hand Excavation and ChemoMechanical Technique, Caries Res 46(6) (2012) 561-567.

[8] N.J. Cochrane, P. Anderson, G.R. Davis, G.G. Adams, M.A. Stacey, E.C. Reynolds, An X-ray Microtomographic Study of Natural White-spot Enamel Lesions, J Dent Res 91(2) (2012) 185-191.

[9] D.P. Clark, C.T. Badea, Micro-CT of rodents: State-of-the-art and future perspectives, Phys Medica 30(6) (2014) 619-634.

[10] N.R. Mohammed, R.J.M. Lynch, P. Anderson, Effects of fluoride concentration on enamel demineralization kinetics in vitro, J Dent 42(5) (2014) 613-618.

[11] G.R. Davis, A.N.Z. Evershed, D. Mills, Quantitative high contrast X-ray microtomography for dental research, J Dent 41(5) (2013) 475-482.

[12] G.R. Davis, J.C. Elliott, X-ray microtomography scanner using time-delay integration for elimination of ring artefacts in the reconstructed image, Nucl Instrum Meth A 394(1-2) (1997) 157-162.

[13] N.R. Mohammed, R.J.M. Lynch, P. Anderson, Inhibitory Effects of Zinc Ions on Enamel Demineralisation Kinetics in vitro, Caries Res 49(6) (2015) 600-605.

[14] N.J. Cochrane, Y. Iijima, P.Y. Shen, Y. Yuan, G.D. Walker, C. Reynolds, C.M. MacRae, N.C. Wilson, G.G. Adams, E.C. Reynolds, Comparative Study of the Measurement of Enamel Demineralization and Remineralization Using Transverse Microradiography and Electron Probe Microanalysis, Microsc Microanal 20(3) (2014) 937-945.

[15] A.N.Z. Evershed, D. Mills, G.R. Davis, Multi-species Beam Hardening Calibration Device for X-ray Microtomography, Proc Spie 8506 (2012) 85061N

[16] G. Davis, D. Mills, 2D beam hardening correction for micro-CT of immersed hard tissue, Developments in X-Ray Tomography X 9967 (2016).

[17] L.A. Feldkamp, L.C. Davis, J.W. Kress, Practical Cone-Beam Algorithm, J Opt Soc Am A 1(6) (1984) 612-619.

[18] X. Liu, K. Laperre, A. Sasov, Practical pseudo-3D registration for large tomographic images, Developments in X-Ray Tomography Ix 9212 (2014).

[19] M.J. Berger, Hubbell, J.H., Seltzer, S.M., Chang, J., Coursey, J.S., Sukumar, R., Zucker, D.S., and Olsen, K., XCOM: Photon Cross Sections Database (version 1.5). <http://physics.nist.gov/xcom>, 2010 (accessed October 16th.2017).

[20] T. Hildebrand, P. Ruegsegger, A new method for the model-independent assessment of thickness in three-dimensional images, J Microsc-Oxford 185 (1997) 67-75. 